\title{
SYMMETRY ANALYSIS OF AND FIRST INTEGRALS FOR THE CONTINUUM HEISENBERG SPIN CHAIN
}

\author{
M. C. $\mathrm{NUCCI}^{1}$ and P. G. L. LEACH ${ }^{2}$
}

(Received 24 June, 2000; revised 22 May, 2001)

\begin{abstract}
Daniel et al. [6] analysed the singularity structure of the continuum limit of the onedimensional anisotropic Heisenberg spin chain in a transverse field and determined the conditions under which the system is nonintegrable and exhibits chaos. We investigate the governing differential equations for symmetries and find the associated first integrals. Our results complement the results of Daniel et al.
\end{abstract}

\section{Introduction}

Towards the end of the nineteenth century two very significant developments occurred in the field of differential equations. The first of them was the systematisation of the formerly ad hoc techniques for the integration of differential equations through the concept of symmetries which was developed by Lie in a series of lengthy papers and books [15-17]. The second, which was an outcome of the study of complex functions initiated by Cauchy, was the analysis of the singularities of the solutions of ordinary differential equations. Generally known as the Painlevé analysis these investigations commenced with the work of Kovalevski on the rotation of a rigid body with one point fixed [12], received many contributions from Painlevé [18-20] and was continued by Gambier [8], Chazy [3] and Bureau [1,2] even up to the present day with the works of Kruskal and Clarkson [13], Cosgrove [5] and Conte [4]. The singularity analysis of partial differential equations was developed by Weiss $e$ t al. [21] and greatly simplified by an Ansatz of Kruskal [11]. One of the outstanding questions in the discussion

\footnotetext{
'Dipartimento di Matematica e Informatica, Università di Perugia, 06123 Perugia, Italy; e-mail: nucci@unipg.it.

${ }^{2}$ GEODYSYC, Department of Mathematics, University of the Aegean, Karlovassi 83200 , Greece.

Permanent address: School of Mathematical and Statistical Sciences, University of Natal, Durban 4041, Republic of South Africa.
}

(C) Australian Mathematical Society 2002, Serial-fee code 1446-1811/02 
of the integrability of differential equations is the connection between symmetry and singularity. There has been some progress along these lines [7, 14], but it would seem that the twentieth century has come to pass without a definite answer to this question.

Daniel et al. [6] analysed the singularity structure of the continuum limit of the one-dimensional anisotropic Heisenberg spin chain in a transverse field with the Hamiltonian

$$
H=-J \sum \mathbf{S}_{i} \cdot \mathbf{S}_{i+1}+A \sum\left(S_{i}^{z}\right)^{2}-\mu \mathbf{B} \cdot \sum \mathbf{S}_{i},
$$

where $\mathbf{S}_{i}=\left(S_{i}^{x}, S_{i}^{y}, S_{i}^{z}\right), i=1, \ldots, N$, are three-component unit vectors with only nearest neighbour interactions, $J>0$ is the pair interaction parameter, $A$ is the anisotropy parameter ( $>0$, easy plane; $<0$, easy axis) and $\mu=g \mu_{B}$ is the gyromagnetic ratio. The corresponding equation of motion in the continuum limit becomes the Landau-Lipshitz equation

$$
\mathbf{S}_{t}=\mathbf{S} \times\left[\mathbf{S}_{z z}-2 A(\mathbf{S} \cdot \mathbf{n}) \mathbf{n}+\mu \mathbf{B}\right],
$$

where the subscripts $t$ and $z$ denote partial differentiation and $\mathbf{S}(t, z)=\left(S^{x}, S^{y}, S^{z}\right)$, $|\mathbf{S}|=1$ and $\mathbf{n}=(0,0,1)$. Daniel $e t$ al. performed the singularity analysis of the system

$$
\begin{aligned}
(1+F G)\left[i F_{t}+F_{z z}+\mu B\left(1-F^{2}\right) / 2\right]-2 G F_{z}^{2}+2 A F(1-F G) & =0, \\
(1+F G)\left[-i G_{t}+G_{z z}+\mu B\left(1-G^{2}\right) / 2\right]-2 F G_{z}^{2}+2 A G(1-F G) & =0,
\end{aligned}
$$

where the transverse field is given by $\mathbf{B}=(B, 0,0), B$ is a constant and

$$
F=\frac{S^{x}+i S^{y}}{1+S^{z}} \quad \text { and } \quad G=\frac{S^{x}-i S^{y}}{1+S^{z}} .
$$

From their analysis they concluded that the anisotropic Heisenberg spin chain in a transverse magnetic field is nonintegrable when both of the parameters $A$ and $B$ are nonzero. It becomes integrable when one or other or both of the constants is zero, that is, the anisotropic interaction and/or the external magnetic field is zero. They confirmed their results of the nonintegrability of the system by a numerical examination of the static version of (1) for which they found chaotic patterns.

In this paper we demonstrate that the Lie point symmetry analysis and consequent determination of the first integrals when they can be found leads us to the same conclusion. We recall that the possession of the Painlevé property is a stronger condition for integrability than the possession of a sufficient number of Lie point symmetries and associated first integrals/invariants since it requires the solution to be meromorphic at all movable singularities. 


\section{Formulation of the problem in plane polar coordinates}

Daniel et al. [6] assume $F=P(z)+i Q(z)$ to arrive at the static equations of (1), that is,

$$
\begin{aligned}
& \left(1+P^{2}+Q^{2}\right) \ddot{P}-2 P \dot{P}^{2}+2 P \dot{Q}^{2}-4 \dot{P} Q \dot{Q}+2 A P\left(1-P^{2}-Q^{2}\right) \\
& \quad+(\mu B / 2)\left(1+P^{2}+Q^{2}\right)\left(1-P^{2}+Q^{2}\right)=0 \\
& \left(1+P^{2}+Q^{2}\right) \ddot{Q}-2 Q \dot{Q}^{2}+2 Q \dot{P}^{2}-4 P \dot{P} \dot{Q}+2 A Q\left(1-P^{2}-Q^{2}\right) \\
& \quad-\mu B\left(1+P^{2}+Q^{2}\right) P Q=0,
\end{aligned}
$$

in which we are denoting differentiation with respect to the independent variable $z$ by an overdot.

We can simplify the appearance of (2) and (3) by means of the combinations $P \times(2)+Q \times(3)$ and $P \times(3)-Q \times(2)$ and a change of variables to plane polar coordinates given by

$$
P=r \cos \theta, \quad Q=r \sin \theta
$$

In the new coordinate system the first combination gives the equation

$$
\left(1+r^{2}\right)\left(\ddot{r}-r \dot{\theta}^{2}\right)-2 r \dot{r}^{2}+2 r^{3} \dot{\theta}^{2}+2 A r\left(1-r^{2}\right)+\frac{1}{2} \mu B \cos \theta\left(1-r^{2}\right)\left(1+r^{2}\right)=0
$$

and the second combination gives the equation

$$
\left(1+r^{2}\right)(r \ddot{\theta}+2 \dot{r} \dot{\theta})-4 r^{2} \dot{r} \dot{\theta}-\frac{1}{2} \mu B \sin \theta\left(1+r^{2}\right)^{2}=0 .
$$

Daniel et al. observed that the system (2) and (3) could be written in Hamiltonian form. In terms of the plane polar coordinates the Hamiltonian is

$$
H=\frac{1}{2}\left(1+r^{2}\right)^{2}\left(p_{r}^{2}+\frac{1}{r^{2}} p_{\theta}^{2}\right)+\frac{A r^{2}}{\left(1+r^{2}\right)^{2}}+\frac{1}{2} \mu B \frac{r \cos \theta}{1+r^{2}},
$$

where the canonical momenta are given by

$$
p_{r}=\frac{\dot{r}}{\left(1+r^{2}\right)^{2}} \quad \text { and } \quad p_{\theta}=\frac{r^{2} \dot{\theta}}{\left(1+r^{2}\right)^{2}}
$$

\section{The Lie point symmetries}

The number of Lie point symmetries and their appearance depends upon the presence or absence of the two parameters $A$ and $B$. We distinguish four cases. 
(i) $A=0, B=0$ : There are five Lie point symmetries given by

$$
\begin{array}{ll}
G_{1}=\partial_{z}, & G_{4}=\left(1+r^{2}\right) \cos \theta \partial_{r}+(r-1 / r) \sin \theta \partial_{\theta}, \\
G_{2}=z \partial_{z}, & G_{5}=-\left(1+r^{2}\right) \sin \theta \partial_{r}+(r-1 / r) \cos \dot{\theta} \partial_{\theta}, \\
G_{3}=\partial_{\theta}, &
\end{array}
$$

which have the algebra $A_{2} \oplus A_{3,8}$, where $A_{3,8}$ is in a representation of $s o(2,1)$ rather than $s l(2, R)$.

We note in passing that an alternative representation of $G_{4}$ and $G_{5}$ is

$$
G_{ \pm}= \pm i G_{4}+G_{5}=\mathrm{e}^{ \pm i \theta}\left[ \pm i\left(1+r^{2}\right) \partial_{r}+(r-\dot{1} / r) \partial_{\theta}\right]
$$

(ii) $A \neq 0, B=0$ : In this case the number of Lie point symmetries is reduced to two. They are $G_{1}$ and $G_{3}$ of (6) with the algebra $2 A_{1}$.

(iii) $A=0, B \neq 0$ : Again there are two symmetries. They are $G_{1}$ and $G_{5}$ of (6) which also have the algebra $2 A_{1}$.

(iv) $A \neq 0, B \neq 0$ : In this case there is the single symmetry $G_{1}$ of (6).

The way the symmetry breaking works is quite interesting. There is no introduction of a new symmetry to accommodate the additional terms, such as happens with $\ddot{x}=0$ and $\ddot{x}+x=0$ for example. If an existing symmetry is compatible with the term introduced, it remains. Otherwise it is lost. The loss of the homogeneity symmetry, $G_{2}$, from (6) is not surprising since it really reflects the fact that without the parameters all terms in the equations contain two differentiations with respect to $z$. When the parameter $A$ is introduced, the loss of $G_{4}$ and $G_{5}$ is not surprising as they represent more of an interaction between the radial and angular variables than a purely radial effect. At first sight the persistence of $G_{5}$ when $B$ is introduced while $G_{4}$ is lost strikes one as curious, but eventually we shall see that it makes sense.

\section{First integrals for the case $A=0, B=0$}

A first integral, $I(z, r, \theta, \dot{r}, \dot{\theta})$, associated with a Lie point symmetry $G$ satisfies the two conditions

$$
G^{[1]} I=0 \quad \text { and } \quad \frac{\mathrm{d} I}{\mathrm{~d} z}=0,
$$

where the first condition expresses the requirement that the function be invariant under the action of the first extension of $G$ and the second that the function be a constant in $z$. In this case (4) and (5) become

$$
\begin{aligned}
\left(1+r^{2}\right)\left(\ddot{r}-r \dot{\theta}^{2}\right)-2 r \dot{r}^{2}+2 r^{3} \dot{\theta}^{2} & =0, \\
\left(1+r^{2}\right)(r \ddot{\theta}+2 \dot{r} \dot{\theta})-4 r^{2} \dot{r} \dot{\theta} & =0 .
\end{aligned}
$$


We seek the first integrals associated with $G_{1}=\partial_{z}$. The first condition of (7) gives the associated Lagrange's system

$$
\frac{\mathrm{d} z}{1}=\frac{\mathrm{d} r}{0}=\frac{\mathrm{d} \theta}{0}=\frac{\mathrm{d} \dot{r}}{0}=\frac{\mathrm{d} \dot{\theta}}{0},
$$

which has the obvious invariants $r, \theta, \dot{r}$ and $\dot{\theta}$. The second condition of (7) gives the associated Lagrange's system

$$
\frac{\mathrm{d} r}{\dot{r}}=\frac{\mathrm{d} \theta}{\dot{\theta}}=\frac{\left(1+r^{2}\right) \mathrm{d} \dot{r}}{r\left[2 \dot{r}^{2}-\left(r^{2}-1\right) \dot{\theta}^{2}\right]}=\frac{r\left(1+r^{2}\right) \mathrm{d} \dot{\theta}}{2\left(r^{2}-1\right) \dot{r} \dot{\theta}} .
$$

The combination of the first and fourth terms of (8) gives

$$
0=\frac{\mathrm{d} \dot{\theta}}{\mathrm{d} r}-\frac{2\left(r^{2}-1\right)}{r\left(1+r^{2}\right)} \dot{\theta}
$$

which is easily integrated to give the first integral

$$
I_{1}=\frac{r^{2} \dot{\theta}}{\left(1+r^{2}\right)^{2}} \text {. }
$$

This integral is suggestive of angular momentum.

We may use the integral $I_{1}$ to eliminate $\dot{\theta}$ from the third term of (8) and so, with the first member of (8), we have an equation in just the two variables $r$ and $\dot{r}$. The combination of the two gives

$$
\begin{aligned}
0 & =\dot{r} \frac{\mathrm{d} \dot{r}}{\mathrm{~d} r}-\frac{2 r \dot{r}^{2}}{1+r^{2}}+\frac{r\left(r^{2}-1\right)}{1+r^{2}} \dot{\theta}^{2}, \\
\Leftrightarrow \quad 0 & =\frac{\mathrm{d}\left(\dot{r}^{2}\right)}{\mathrm{d}\left(r^{2}\right)}-\frac{2 \dot{r}^{2}}{1+r^{2}}+\frac{r^{2}-1}{1+r^{2}} \dot{\theta}^{2}=\frac{\mathrm{d}}{\mathrm{d}\left(r^{2}\right)}\left(\frac{\dot{r}^{2}}{\left(1+r^{2}\right)^{2}}\right)+l_{1}^{2}\left(1-\frac{1}{r^{4}}\right)
\end{aligned}
$$

which is easily integrated to give another first integral

$$
I_{2}=\frac{\dot{r}^{2}}{\left(1+r^{2}\right)^{2}}+I_{1}^{2}\left(r^{2}+\frac{1}{r^{2}}\right) .
$$

The structure of the first integral is rendered more attractive by taking the combination $I_{2}+2 I_{1}^{2}$ to give the energy-like integral

$$
E=\frac{\dot{r}^{2}+r^{2} \dot{\theta}^{2}}{\left(1+r^{2}\right)^{2}}
$$

With these two first integrals we may eliminate $\dot{r}$ and $\dot{\theta}$ from the combination of the first and second terms of (8), namely

$$
\mathrm{d} \theta=\frac{\dot{\theta}}{\dot{r}} \mathrm{~d} r
$$


to obtain

$$
\mathrm{d} \theta=\frac{\left(1+r^{2}\right) I_{1} \mathrm{~d} r}{r \sqrt{I_{2} r^{2}-I_{1}^{2}\left(1+r^{4}\right)}}
$$

in which we have used $I_{2}$ instead of $E$ since the resulting expression is simpler. The relationship between the radial distance and the angle is found from the quadrature

$$
\theta-\theta_{0}=\frac{1}{2} \int^{r^{2}} \frac{(1+\eta) \mathrm{d} \eta}{\sqrt{\left(I_{2}^{2} / 4 I_{1}^{4}\right)-1-\left(\eta-\left(I_{2} / 2 I_{1}^{2}\right)\right)^{2}}} .
$$

Since $I_{2}-2 I_{1}^{2}>0$, the integral is real and is easily evaluated by a trigonometric substitution. We obtain [10, 2.551.3, p. 179]

$$
\theta-\theta_{0}=\frac{1}{2}\left\{\phi+2 \arctan \left(\frac{I_{2}}{2 I_{1}^{2}} \tan \frac{\phi}{2}+\sqrt{\frac{I_{2}^{2}}{4 I_{1}^{4}}-1}\right)\right\}
$$

where we have made use of the substitution

$$
\eta=\frac{I_{2}}{2 l_{1}^{2}+\sqrt{\left(I_{2}^{2} / 4 I_{1}^{4}\right)-1} \sin \phi} .
$$

We observe that (11) is not invertible to give $r$ as a function of $\theta$ except locally.

The evolution of the system in $z$ cannot be obtained using $G_{1}$. We turn to $G_{3}$ for which the first condition of (7) gives the associated Lagrange's system

$$
\frac{\mathrm{d} z}{0}=\frac{\mathrm{d} r}{0}=\frac{\mathrm{d} \theta}{1}=\frac{\mathrm{d} \dot{r}}{0}=\frac{\mathrm{d} \dot{\theta}}{0}
$$

which has the obvious invariants $z, r, \dot{r}$ and $\dot{\theta}$. The second condition of (7) gives the associated Lagrange's system

$$
\frac{\mathrm{d} z}{1}=\frac{\mathrm{d} r}{\dot{r}}=\frac{\left(1+r^{2}\right) \mathrm{d} \dot{r}}{r\left[2 \dot{r}^{2}-\left(r^{2}-1\right) \dot{\theta}^{2}\right]}=\frac{r\left(1+r^{2}\right) \mathrm{d} \dot{\theta}}{2\left(r^{2}-1\right) \dot{r} \dot{\theta}} .
$$

Evidently combinations of the second, third and fourth terms of (12) and (13) will give the two integrals $I_{1}$ and $I_{2}$ already obtained. From the first and the second terms we have $\mathrm{d} z=\mathrm{d} r / \dot{r}$ from which, in a manner analogous to that for the calculation of $\theta-\theta_{0}$, we obtain from (9) and (10), that is, the two first integrals $I_{1}, I_{2}$,

$$
\dot{r}=\frac{r^{2}+1}{r} \sqrt{I_{2} r^{2}-I_{1}^{2}\left(r^{4}+1\right)}
$$


which can be integrated through MAPLE 6 to give

$$
2 \sqrt{I_{2}+2 I_{1}^{2}}\left(z-z_{0}\right)=\arctan \left(\frac{\left(I_{2}+2 I_{1}^{2}\right)\left(r^{2}-1\right)}{2 \sqrt{\left(I_{2}+2 I_{1}^{2}\right)\left(I_{2} r^{2}-I_{1}^{2}\left(r^{4}+1\right)\right)}}\right) .
$$

It is possible to invert the result of the integration to give

$$
r^{2}=\frac{\sqrt{I_{2}+2 I_{1}^{2}} \pm \sqrt{I_{2}-2 I_{1}^{2}} \sin W}{\sqrt{I_{2}+2 I_{1}^{2}} \mp \sqrt{I_{2}-2 I_{1}^{2}} \sin W}
$$

where $W=2 \sqrt{I_{2}+2 I_{1}^{2}}\left(z-z_{0}\right)$. Evidently the motion is periodic in $z$.

\section{First integrals for the case $A \neq 0, B=0$}

For this case (4) and (5) become

$$
\begin{aligned}
\left(1+r^{2}\right)\left(\ddot{r}-r \dot{\theta}^{2}\right)-2 r \dot{r}^{2}+2 r^{3} \dot{\theta}^{2}+\frac{2 A r\left(1-r^{2}\right)}{1+r^{2}} & =0, \\
\left(1+r^{2}\right)(r \ddot{\theta}+2 \dot{r} \dot{\theta})-4 r^{2} \dot{r} \dot{\theta} & =0 .
\end{aligned}
$$

We commence with $G_{1}=\partial_{z}$. The first condition of (7) gives the obvious invariants $r, \theta, \dot{r}$ and $\dot{\theta}$. The second condition of (7) gives the associated Lagrange's system

$$
\frac{\mathrm{d} r}{\dot{r}}=\frac{\mathrm{d} \theta}{\dot{\theta}}=\frac{\left(1+r^{2}\right) \mathrm{d} \dot{r}}{r\left[2 \dot{r}^{2}-\left(r^{2}-1\right) \dot{\theta}^{2}\right]-2 A r\left(1-r^{2}\right)}=\frac{r\left(1+r^{2}\right) \mathrm{d} \dot{\theta}}{2\left(r^{2}-1\right) \dot{r} \dot{\theta}} .
$$

From the combination of the first and fourth terms of (14) we again obtain the first integral $I_{1}=r^{2} \dot{\theta} /\left(1+r^{2}\right)^{2}$.

Using $I_{1}$ and the first and third terms of (14) we obtain

$$
0=\frac{\mathrm{d}}{\mathrm{d}\left(\dot{r}^{2}\right)}\left(\frac{r^{2}}{\left(1+r^{2}\right)^{2}}\right)+I_{1}^{2}\left(1-\frac{1}{r^{4}}\right)+2 A \frac{1-r^{2}}{\left(1+r^{2}\right)^{3}}
$$

which is easily integrated to give

$$
I_{2}=\frac{\dot{r}^{2}}{\left(1+r^{2}\right)^{2}}+I_{1}^{2}\left(r^{2}+\frac{1}{r^{2}}\right)+\frac{2 A r^{2}}{\left(1+r^{2}\right)^{2}}
$$

This is obviously the generalisation of (10). Again we take the combination $I_{2}+2 I_{1}^{2}$ to obtain the energy-like integral

$$
E=\frac{\dot{r}^{2}+r^{2} \dot{\theta}^{2}}{\left(1+r^{2}\right)^{2}}+\frac{2 A r^{2}}{\left(1+r^{2}\right)^{2}} .
$$


From the first and second terms of (14) we obtain

$$
\mathrm{d} \theta=\frac{\left(1+r^{2}\right) I_{1} \mathrm{~d} r}{r \sqrt{I_{2} r^{2}-I_{1}^{2}\left(1+r^{4}\right)-2 A r^{4}}}
$$

after the elimination of $\dot{r}$ and $\dot{\theta}$. From this we obtain the relationship between $\theta$ and $r$. As a function of $r$ the integrand is a complication on the square root of a quartic polynomial which one presumes can be expressed as a collection of elliptic integrals and which one would not expect to be able to invert to obtain the orbit equation in the standard form of $r(\theta)$.

With the symmetry $\partial_{\theta}$ we again obtain $I_{1}$ and $I_{2}$ as two of the characteristics of the second condition of (7). The third characteristic gives the evolution in $z$ as the quadrature

$$
z-z_{0}=\int^{r^{2}} \frac{\mathrm{d} \eta}{\sqrt{I_{2} \eta(1+\eta)^{2}-I_{1}^{2}\left(\eta^{2}+1\right)(1+\eta)^{2}-2 A \eta^{2}}}
$$

which will be an expression of somewhat less complexity than that obtained by the quadrature of (15), but still not invertible.

\section{First integrals for the case $A=0, B \neq 0$}

In this case we have

$$
\begin{aligned}
\left(1+r^{2}\right)\left(\ddot{r}-r \dot{\theta}^{2}\right)-2 r \dot{r}^{2}+2 r^{3} \dot{\theta}^{2}+\frac{1}{2} \mu B \cos \theta\left(1-r^{2}\right)\left(1+r^{2}\right) & =0 \\
\left(1+r^{2}\right)(r \ddot{\theta}+2 \dot{r} \dot{\theta})-4 r^{2} \dot{r} \dot{\theta}+\frac{1}{2} \mu \sin \theta\left(1+r^{2}\right)^{2} & =0
\end{aligned}
$$

The symmetry $\partial_{z}$ has the invariants $r, \theta, \dot{r}$ and $\dot{\theta}$. The second condition of (7) gives the associated Lagrange's system

$$
\begin{aligned}
\frac{\mathrm{d} r}{\dot{r}}=\frac{\mathrm{d} \theta}{\dot{\theta}} & =\frac{\mathrm{d} \dot{r}}{\frac{r}{1+r^{2}}\left[2 \dot{r}^{2}-\left(r^{2}-1\right) \dot{\theta}^{2}\right]-\frac{1}{2} \mu B \cos \theta\left(1-r^{2}\right)} \\
& =\frac{\mathrm{d} \dot{\theta}}{\frac{2\left(r^{2}-1\right)}{r\left(1+r^{2}\right)} \dot{r} \dot{\theta}-\frac{1}{2} \mu B \sin \theta\left(1+r^{2}\right)}
\end{aligned}
$$

This system of first-order equations is far more difficult to solve than the comparable systems in the first two cases. However, one can proceed to make combinations which eliminate terms in the denominator of the third and fourth terms in (16) and then to 
combine them to obtain the integral. The combination is

$$
\begin{aligned}
& \mathrm{d}\left(\frac{1}{\left(1+r^{2}\right)^{2}}\right) \dot{r}^{2}+\frac{1}{\left(1+r^{2}\right)^{2}} \mathrm{~d}\left(\dot{r}^{2}\right)+\mathrm{d}\left(\dot{\theta}^{2}\right) \frac{r^{2}}{\left(1+r^{2}\right)^{2}} \\
& \quad+\dot{\theta}^{2} \frac{2 r\left(1-r^{2}\right)}{\left(1+r^{2}\right)^{3}} \mathrm{~d} r+\mu B \cos \theta \frac{1-r^{2}}{r\left(1+r^{2}\right)^{2}} \mathrm{~d} r-\mu B \frac{r}{1+r^{2}} \sin \theta \mathrm{d} \theta
\end{aligned}
$$

and the integral is

$$
I_{1}=\frac{\dot{r}^{2}+r^{2} \dot{\theta}^{2}}{\left(1+r^{2}\right)^{2}}+\mu B \frac{r \cos \theta}{1+r^{2}} .
$$

This is a bit of a piecemeal approach, but that is the way the integral comes together. We note that we obtain the energy-like integral immediately, probably because there is no angular momentum-type integral due to the presence of $\theta$ in the equations of motion.

The rationale behind the combinations used to obtain (18) was that of finding a first integral quadratic in the velocities. We observe that a combination of the third and fourth terms of (17) can be made which eliminates the terms which are not quadratic in $\dot{r}$ and $\dot{\theta}$. We can then eliminate the quadratic terms using the first and second terms of (17) to obtain zero in the denominator. By a happy happenstance the numerator is exact and so we obtain a second integral. The combination is

$$
\begin{aligned}
& \frac{\sin \theta}{1+r^{2}} \mathrm{~d} \dot{r}-\frac{\dot{r}\left(r^{2}-1\right)}{\left(1+r^{2}\right)^{2}} \cos \theta \mathrm{d} \dot{\theta}+\frac{r\left(r^{2}-1\right)}{\left(1+r^{2}\right)^{2}} \sin \theta \dot{\theta} \mathrm{d} \theta \\
& \quad+\frac{\cos \theta}{1+r^{2}} \dot{r} \mathrm{~d} \theta-\frac{2 r}{\left(1+r^{2}\right)^{2}} \sin \theta \dot{r} \mathrm{~d} r+\frac{1-6 r^{2}+r^{4}}{\left(1+r^{2}\right)^{3}} \cos \theta \dot{\theta} \mathrm{d} r
\end{aligned}
$$

which indicates the term by term elimination subsequent to the combination of the third and the fourth terms of (17). Fortunately the integral does not look quite as horrendous as (19) could suggest. It is

$$
I_{2}=\frac{\dot{r} \sin \theta}{1+r^{2}}+\frac{r\left(1-r^{2}\right) \dot{\theta} \cos \theta}{\left(1+r^{2}\right)^{2}} .
$$

This integral is reminiscent of the conservation of momentum and is probably the generalisation of the angular momentum-like integral found in the cases for which $B=0$.

We may use (18) and (20) to eliminate $\dot{r}$ and $\dot{\theta}$ from (16) to obtain a first-order equation in $r$ and $\theta$. However, it is so complicated that one has serious doubts about the existence of a closed-form solution. We simply give the equation which is

$$
\frac{1}{r} \frac{\mathrm{d} r}{\mathrm{~d} \theta}=\frac{\left(1+r^{2}\right) I_{2} \sin \theta \pm\left(1-r^{2}\right)^{2} Q}{I_{2}\left(1-r^{2}\right) \pm\left(1-r^{2}\right)\left(1+r^{2}\right) \sin \theta Q},
$$


where

$$
Q=\sqrt{\left[\frac{1}{\left(1+r^{2}\right)^{2}}+\frac{\sin ^{2} \theta}{\left(1-r^{2}\right)^{2}}\right]\left[2 I_{1}-\frac{\mu B r \sin \theta}{1+r^{2}}\right]-\frac{I_{2}^{2}}{\left(1-r^{2}\right)^{2}}}
$$

Nevertheless the existence of the two independent first integrals guarantees integrability since the system is Hamiltonian.

\section{First integrals for the case $A \neq 0, B \neq 0$}

We now have the full system

$$
\begin{aligned}
& \left(1+r^{2}\right)\left(\ddot{r}-r \dot{\theta}^{2}\right)-2 r \dot{r}^{2}+2 r^{3} \dot{\theta}^{2}+2 A r\left(1-r^{2}\right)+\frac{1}{2} \mu B \cos \theta\left(1-r^{2}\right)\left(1+r^{2}\right)=0, \\
& \left(1+r^{2}\right)(r \ddot{\theta}+2 \dot{r} \dot{\theta})-4 r^{2} \dot{r} \dot{\theta}-\frac{1}{2} \mu B \sin \theta\left(1+r^{2}\right)^{2}=0
\end{aligned}
$$

and the single Lie point symmetry $\partial_{z}$ with the characteristics $r, \theta, \dot{r}$ and $\dot{\theta}$. The first integrals are to be found from

$$
\begin{aligned}
\frac{\mathrm{d} r}{\dot{r}}=\frac{\mathrm{d} \theta}{\dot{\theta}} & =\frac{\mathrm{d} \dot{r}}{\frac{r}{1+r^{2}}\left[2 \dot{r}^{2}-\left(r^{2}-1\right) \dot{\theta}^{2}\right]-2 A r\left(\frac{1-r^{2}}{1+r^{2}}\right)-\frac{1}{2} \mu B \cos \theta\left(1-r^{2}\right)} \\
& =\frac{\mathrm{d} \dot{\theta}}{\frac{2\left(r^{2}-1\right)}{r\left(1+r^{2}\right)} \dot{r} \dot{\theta}-\frac{1}{2} \mu B \sin \theta\left(1+r^{2}\right)}
\end{aligned}
$$

and we find the obvious one, the Hamiltonian,

$$
I_{1}=\frac{1}{2} \frac{\dot{r}^{2}+r^{2} \dot{\theta}^{2}}{\left(1+r^{2}\right)^{2}}+\frac{A r^{2}}{\left(1+r^{2}\right)^{2}}+\frac{1}{2} \mu B \frac{r \cos \theta}{1+r^{2}} .
$$

The calculation of any other characteristics from (21) is by no means obvious. This in itself is not a sure indication of nonintegrability since our search for symmetries has been confined to Lie point symmetries. However, in the paper by Daniel et al. [6] there is evidence, both from the singularity analysis of the Painlevé test and numerical experiments, of the nonintegrability of the original system, (2) and (3). In the light of those results we would not expect to be able to find any symmetry which would be of use in the reduction of the order of the system to an autonomous two-dimensional system which is always integrable. (One can always find an infinite number of symmetries for any differential equation [9].) 


\section{Acknowledgements}

This paper is dedicated to Martin Kruskal and his accomplishments on the occasion of his 75th birthday.

MCN is supported in part by Fondi MURST (Cofin 97: Metodi e applicazioni di equazioni differenziali ordinarie). PGLL thanks the Dipartimento di Matematica e Informatica, Università di Perugia, for its kind hospitality during the period when this work was undertaken and acknowledges the continued support of the National Research Foundation of South Africa and the University of Natal.

\section{References}

[1] F. J. Bureau, "Differential equations with fixed critical points", Ann. Mat. Pura Appl. (4) 64 (1964) 229-364.

[2] F. J. Bureau, "Differential equations with fixed critical points", Ann. Mat. Pura Appl. (4) 66 (1964) $1-116$.

[3] J. Chazy, "Sur les équations différentielles du troisième ordre et d'ordre supérieur dont l'integrale générale est à ses points critiques fixes", Acta Math. 34 (1911) 317-385.

[4] R. Conte, "Singularities of differential equations and integrability", in Introduction to Methods of Complex Analysis and Geometry for Classical Mechanics and Nonlinear Waves (eds. D. Benest and Frœschlé), (Éditions Frontières, Gif-sur-Yvette, 1994) 49-143.

[5] C. M. Cosgrove, "All binomial-type Painlevé equations of the second order and degree three or higher", Stud. Appl. Math. 89 (1993) 95-151.

[6] M. Daniel, M. D. Kruskal, M. Lakshmanan and K. Nakamura, "Singularity structure analysis of the continuum Heisenberg spin chain with anisotropy and transverse field: nonintegrability and chaos", J. Math. Phys. 33 (1992) 771-776.

[7] M. R. Feix, C. Géronimi, L. Cairó, P. G. L. Leach, R. L. Lemmer and S. É. Bouquet, "On the singularity analysis of ordinary differential equations invariant under time translation and rescaling", J. Phys. A: Math. Gen. 30 (1997) 7437-7461.

[8] B. Gambier, "Sur les équations différentielles du second ordre et du premier degré dont l'intégrale générale est à points critiques fixes", Acta Math. 33 (1909) 1-55.

[9] K. S. Govinder and P. G. L. Leach, "The nature and uses of symmetries of ordinary differential equations", South African J. Sci. 92 (1996) 23-28.

[10] I. S. Gradshteyn and I. M. Ryzhik, Tables of Integrals, Series and Products, 5th ed. (Academic Press, San Diego, 1994).

[11] M. Jimbo, M. D. Kruskal and T. Miwa, "Painlevé test for the self-dual Yang-Mills equation", Phys. Lett. A 92 (1982) 59-60.

[12] S. V. Kovalevski, "Sur le problème de la rotation d'un corps solide autor d'un point fixe", Acta Math. 12 (1889) 177-232.

[13] M. D. Kruskal and P. A. Clarkson, "The Painlevé-Kovalevski and poly-Painlevé tests for integrability", Stud. Appl. Math. 86 (1992) 87-165.

[14] R. L. Lemmer and P. G. L. Leach, "The Painlevé test, hidden symmetries and the equation $y^{\prime \prime}+y y^{\prime}+k y^{3}=0 "$, J. Phys. A: Math. Gen. 26 (1993) 5017-5024.

[15] S. Lie, Differentialgleichungen (Chelsea, New York, 1967).

[16] S. Lie, Theorie der Transformationsgruppen, Volume I, II and III (Chelsea, New York, 1970). 
[17] S. Lie, Geometrie der Berühstransformationen (Chelsea, New York, 1977).

[18] P. Painlevé, "Mémoire sur les équations différentielles dont l'intégrale générale est uniforme", Bull. Soc. Math. France 28 (1900) 201-261.

[19] P. Painlevé, "Sur les singularités essentielles des équations différentielles", C. R. Acad. Sci. Paris 133 (1901) 910-913.

[20] P. Painlevé, "Sur les équations différentielles du second ordre et d'ordre supérieur dont l'intégrale générale est uniforme", Acta Math. 25 (1902) 1-85.

[21] J. Weiss, M. Tabor and G. Carnevale, "The Painlevé property for partial differential equations", $J$. Math. Phys. 24 (1983) 522-526. 